\title{
Atomic transport and chemical reaction in TiN/Ti nanolayers on plasma nitrided steel
}

\author{
C. Aguzzoli • E.K. Tentardini · C.A. Figueroa • \\ C. Kwietniewski • L. Miotti · I.J.R. Baumvol
}

Published online: 29 July 2008

(C) Springer-Verlag 2008

\section{Erratum to: Appl Phys A}

DOI 10.1007/s00339-008-4758-x

Due to a processing error a mistake occurred in the author affiliations. The corrected affiliations are given below.

The online version of the original article can be found under doi:10.1007/s00339-008-4758-x.

C. Aguzzoli · E.K. Tentardini · C.A. Figueroa - L. Miotti .

I.J.R. Baumvol

Universidade de Caxias do Sul, CCET, Av. Francisco G. Vargas

1130, 95070-560 Caxias do Sul RS, Brazil

\section{Kwietniewski}

Programa de Pós-Graduação em Engenharia de Minas,

Metalúrgica e de Materiais-PPGEM, Universidade Federal

do Rio Grande do Sul, 91501-970 Porto Alegre RS, Brazil

L. Miotti · I.J.R. Baumvol ( $\varangle)$

Instituto de Fisica, Universidade Federal do Rio Grande do Sul, 91501-970 Porto Alegre RS, Brazil

e-mail: israel@if.ufrgs.br 